\title{
Anesthetized Intraperitoneal Lethal Injection and Exsanguination Euthanasia
}

National Cancer Institute

\section{Source}

National Cancer Institute. Anesthetized Intraperitoneal Lethal Injection and

Exsanguination Euthanasia. NCI Thesaurus. Code C116223.

A method of euthanasia whereby a subject is anesthetized, a lethal chemical is administered by intraperitoneal injection and the body is drained of blood. 